\title{
Neumomediastino secundario a efecto Macklin. Reporte de un caso
}

\author{
Gil-Vargas $\mathrm{M}^{1}$, González-Rodríguez LE², León-Aguilar VH³
}

\begin{abstract}
Resumen
El neumomediastino secundario es la presencia de aire en el espacio mediastinal procedente de bronquios, alvéolos o de una ruptura esofágica, que viaja a través de las vainas vasculares y los planos tisulares hacia el espacio mediastinal; puede ser categorizado como espontáneo, traumático o secundario. El neumomediastino secundario es considerado como una entidad poco frecuente en pediatría, de incidencia muy variable. En la edad pediátrica se asocia a crisis asmática y a infecciones de la vía respiratoria, traumatismos, vómitos, esfuerzos intensos, estados de ansiedad con hiperventilación y el consumo de drogas. Presentamos el caso de una niña de 4 años de edad previamente sana, con neumomediastino secundario; no se documentaron posibles mecanismos como traumatismo o procedimientos o instrumentaciones en el tórax en su fisiopatología, se asoció a un acceso de tos, con cuadro de neumonía adquirida en la comunidad. Se confirmo por radiografías de tórax y cuello. El cuadro fue de corta evolución y requirió breve tratamiento intrahospitalario. En la tomografía computada de tórax realizada al ingreso se observó el aire migrando por las vainas peribroncovasculares rumbo al mediastino ("efecto Macklin").
\end{abstract}

PALABRAS CLAVE: tos, neumomediastino secundario, efecto Macklin, reporte de caso.

\section{Pneumomediastinum secondary to Macklin effect. Case report.}

\author{
Gil-Vargas $\mathrm{M}^{1}$, González-Rodríguez LE², León-Aguilar VH³
}

\begin{abstract}
Pneumomediastinum is the presence of air in the mediastinal space, originated in bronchi, alveoli or esophageal rupture, which runs through the vascular tissue pods and towards the mediastinal space. Pneumomediastinum is classified as spontaneous or traumatic. Secondary pneumomediastinum is considered an unusual entity in children, with very variable incidence. It is associated with acute asthma airway infections, trauma, vomiting, intense efforts, anxiety with hyperventilation and drug use. This is a case report of a previously healthy, 4 year old female toddler with secondary pneumome-
\end{abstract}

${ }^{1}$ Médico Cirujano Pediatra.

${ }^{2}$ Médico residente de tercer año de Pediatría. ${ }^{3}$ Médico Neumólogo-Pediatra, Adscrito al Servicio de Pediatría.

Recibido: 22 de agosto del 2016

Aceptado: 2 de diciembre del 2016

Correspondencia

Dr. Manuel Gil Vargas

gilvm@yahoo.com

Este artículo debe citarse como Gil-Vargas M, González-Rodríguez LE, León-Aguilar VHNeumomediastino secundario a efecto Macklin. Reporte de un caso. Acta Pediatr Mex. 2017;38(5):317-321. 
diastinum, without trauma or thoracic procedures or instrumentation in child's pathophysiology associated with cough, with communityacquired pneumonia. It was confirmed by chest and neck radiographs. The pathology was of short duration and required treatment during a short time in the hospital. According to the chest CT scan performed at admission, air was observed migrating from the perivascular bronchial sheaths to the mediastinum ("Macklin effect").

KEYWORDS: cough; secondary pneumomediastinum; Macklin effect; case report
Correspondence Dr. Manuel Gil Vargas gilvm@yahoo.com

\section{INTRODUCCIÓN}

El neumomediastino o enfisema mediastínico se define como la presencia de aire en el mediastino, puede ser traumático, iatrogénico (factores frecuentes) o espontáneo. ${ }^{1,2}$ En niños y adultos el neumomediastino es una patología poco frecuente (aproximadamente de $1 / 800$ a $1 / 42,000$ consultas a urgencias) relacionada con traumatismos en la vía aeréa, ${ }^{3}$ rupturas esofágicas por aumentos de la presión intraabdominal (vómitos), el llamado síndrome de Boerhaave de alta morbimortalidad, ${ }^{4}$ crisis asmática y a infecciones de la vía respiratoria, traumatismos, vómitos, esfuerzos intensos, estados de ansiedad con hiperventilación o espontáneo, en aquellos casos que no presentan relación con trauma o lesión iatrogénica. ${ }^{1,2,5}$

En 1939 Charles Macklin reportó el proceso fisiopatológico en el que la ruptura alveolar es la responsable del neumomediastino. ${ }^{6}$ Su descripción está basada en la sucesión de tres eventos importantes: a) ruptura alveolar por hiperinsuflación de la vía aérea; b) formación de burbujas de aire que migran por las vainas de las arterias y bronquios; c) burbujas de aire que disecan el mediastino por el tejido conectivo y que, dependiendo de la fuerza y volumen de la insuflación bronquial, pueden llegar al cuello, axila o retroperitoneo. ${ }^{1,7}$

\section{REPORTE DE CASO}

Niña de 4 años de edad que ingresó al servicio de urgencias pediátricas, referida con el diagnóstico de neumonía adquirida en la comunidad. Antecedentes personales patológicos: cursó con bronquiolitis al año y medio de edad, recibió tratamiento ambulatorio, refiere la madre que desde ese entonces ha cursado con infecciones de vía aérea recurrentes. Esquema nacional de vacunación completo acorde con la edad.

Inició su padecimiento actual dos días antes del ingreso con tos no productiva, no disneizante, no cianotizante, no emetizante. En 24 horas refirió la madre mayor exacerbación de la tos, siendo ésta ya de tipo productiva, no disneizante, no cianotizante, no emetizante. Al día siguiente, de manera súbita, presentó datos de dificultad respiratoria y enfisema subcutáneo en región cervical y torácica, por lo que fue enviada a nuestra unidad hospitalaria. Ingresó con deterioro en estado neurológico y datos de dificultad respiratoria, aleteo nasal, tiro intercostal marcado, disociación toraco-abdominal visible, por lo que se realizó intubación orotraqueal.

A la exploración física con los siguientes signos vitales: presión arterial 139/53 mmHg, frecuencia cardiaca 129 latidos por minuto, frecuencia respiratoria 32 respiraciones por minuto, temperatura $36^{\circ} \mathrm{C}$. 
Reporte de exámenes de laboratorio: hemoglobina $12.2 \mathrm{~g} / \mathrm{dL}$, hematocrito $35.3 \%$, leucocitos de $2.5610^{3} / \mathrm{mm} 3$, linfocitos $20.7 \%$, monocitos $7.4 \%$, neutrófilos $71.5 \%$, plaquetas $24410^{3 /}$ $\mathrm{mm} 3$. Gasometría arterial con pH 7.27, pCO2 $45 \mathrm{mmHg}$, pO2 $56 \mathrm{mmHg}$, lactato $0.6 \mathrm{mmol} / \mathrm{L}$, HCO3 $20.1 \mathrm{mmol} / \mathrm{L}$, EB $-6.2 \mathrm{mmol} / \mathrm{L}, \mathrm{SO} 2$ 84\%.

En las imágenes radiológicas: aumento de volumen a nivel de la región cervical, bilateral, que se extendía a la parte anterior del tórax, con crepitación a la palpación.

Radiografía de tórax postero-anterior, radiografía lateral de cuello (Figuras 1-3) donde se corroboró aire en tejido celular subcutáneo a nivel del cuello y región superior de tórax, no se observó neumotórax.

Se realizó tomografía computarizada de tórax que mostró neumomediastino y enfisema subcutáneo (Figura 4).

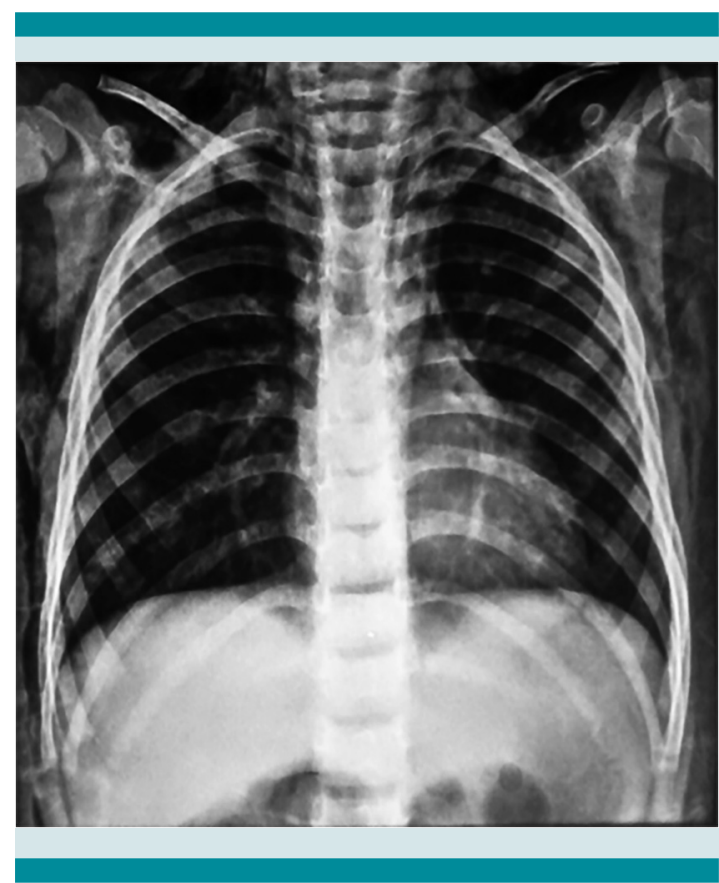

Figura 1. Radiografía antero-posterior previa a intubación.

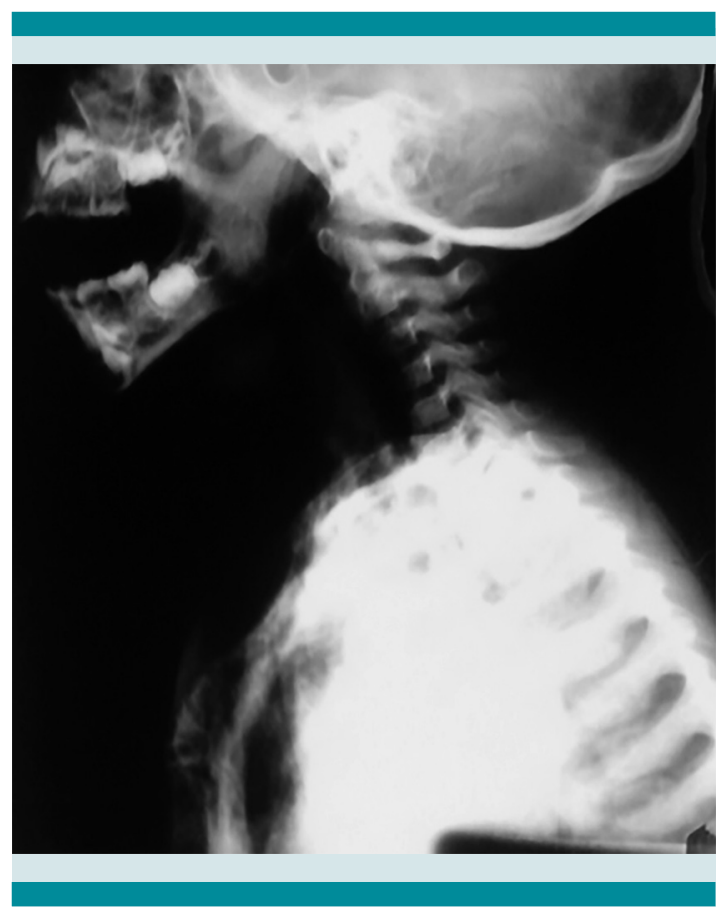

Figura 2. Radiografía lateral de cuello.

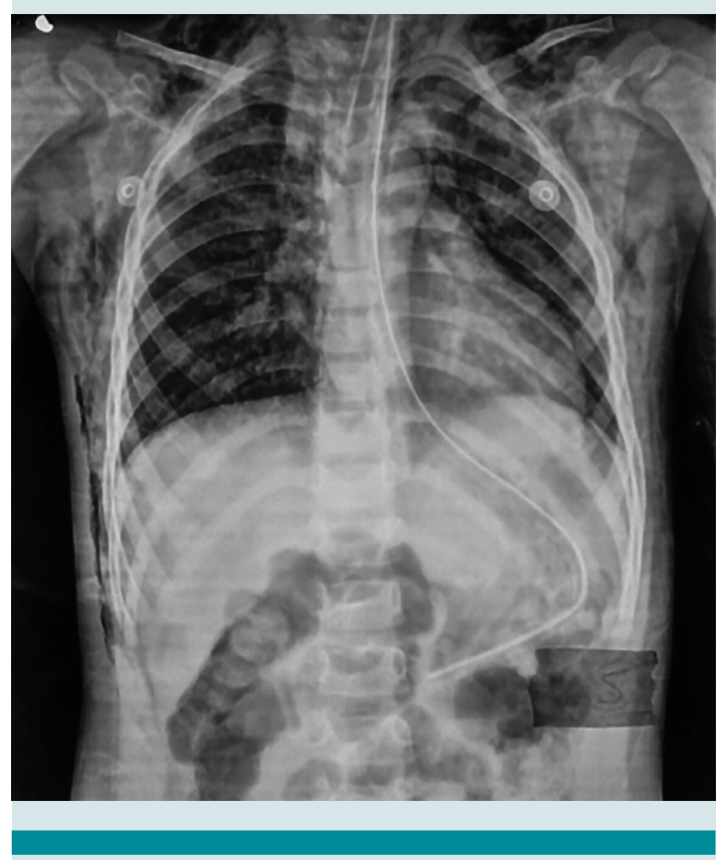

Figura 3. Radiografía antero-posterior posterior a intubación. 


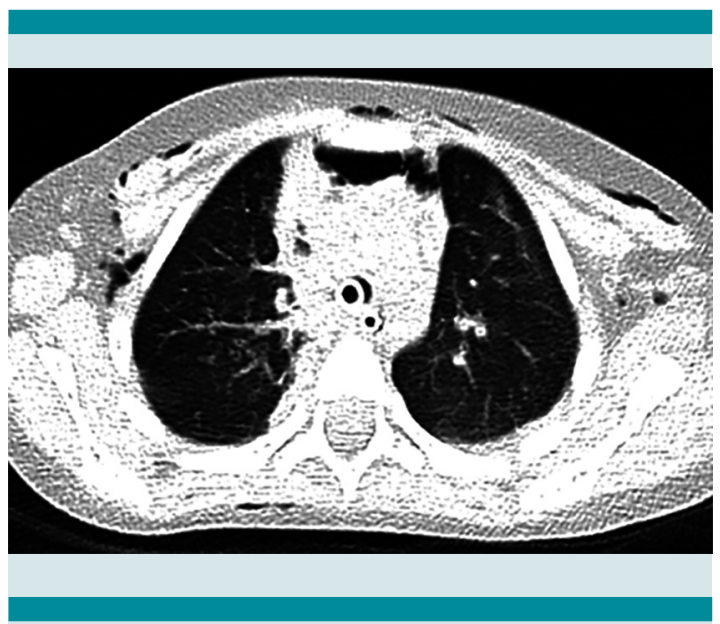

Figura 4. Tomografía axial computarizada simple de tórax.

Con lo anterior, se llegó al diagnóstico de neumomediastino secundario a efecto Macklin. Permaneció durante 24 horas con ventilación mecánica. Logrando extubación programada sin complicaciones. Se otorgó tratamiento con ceftriaxona 75 mgkgdía y vancomicina 40 mgkgdía. Apoyo con oxígeno suplementario.

La paciente se recuperó por completo y egresó por mejoría a los ocho días continuando tratamiento en domicilio.

\section{DISCUSIÓN}

El efecto Macklin fue descrito en 1939 por el Dr. Charles Macklin y explica la etiología de la fuga aérea. El modelo experimental original en el que se validó este mecanismo de lesión consistió en hiperinflación de pulmones de gatos, en los que el gradiente de presión generado entre el alvéolo y el intersticio pulmonar era el principio de la ruptura alveolar y la fuga aérea, lo que da origen al enfisema intersticial. Posteriormente, Marchand describió que la inyección de líquido a elevada presión en el espacio pretraqueal de cadáveres humanos tenía continuidad entre el mediastino y los planos faciales peribronquiales, que fue el fundamento experimental para que Cyrlak demostrara que las fascias periarteriales, perivenosas y peribronquiales tienen continuidad y son una estructura común, lo que explica el patrón de distribución de la fuga aérea en los compartimentos cervical, torácico y abdominal. ${ }^{8}$

Las causas más frecuentes de neumomediastino secundario en niños son: a) Condiciones médicas: asma, fibrosis quística, infecciones del tracto respiratorio superior e inferior, enfermedad por reflujo gastro-esofágico, atragantamiento, enfermedades reumatológicas. b) Maniobras respiratorias: maniobras de Valsalva, vómitos, tos, Ilanto, grito, entrenamiento agotador, ejercicios centrífugos, hipernea, inhalación de helio, inhalación de drogas ilícitas, gases irritantes. c) Condiciones quirúrgicas: aspiración de cuerpo extraño, ruptura espontánea de esófago, perforación gástrica, ruptura de recto. d) latrogénicas: reanimación cardiopulmonar, extracción dentaria, maniobra de Heimlich, test de función pulmonar. $^{9-13}$

El neumomediastino secundario puede distinguirse asimismo en primario, cuando no existe enfermedad subyacente que podría predisponer al escape de aire y secundario cuando existe enfermedad de base predisponente como el asma o la fibrosis quística. ${ }^{9}$

El enfisema intersticial tiene tres presentaciones: ${ }^{8}$

- Enfisema intersticial agudo.

- Enfisema intersticial persistente, localizado a uno o más lóbulos.

- Enfisema intersticial difuso persistente.

El diagnóstico de esta entidad se basa en hallazgos clínicos, radiográficos en tórax, confirmación del efecto Macklin por tomografía computada, 
Gil-Vargas $\mathbf{M}$ et al. Neumomediastino secundario a efecto Macklin

así como broncoscopia y endoscopia para descartar lesiones traqueobronquiales y esofágicas como la causa del enfisema mediastinal. ${ }^{6,14}$

\section{CONCLUSIÓN}

Se comunicó el caso de una paciente de 4 años de edad con un cuadro clínico de enfisema subcutáneo espontáneo con neumomediastino. El cuadro clínico se consideró compatible con neumomediastino secundario a efecto Macklin.

\section{REFERENCIAS}

1. Bilkis Manuel D., Monteverde Ezequiel. Efecto Macklin en el neumomediastino espontáneo pediátrico. Arch Argent Pediatr 2010;108(2):33-36.

2. Aláez A, Galindo J, Páramo J, et al. Un caso de neumomediastino espontáneo que simula un síndrome de Boerhaave. Rev Chil Cir. 2009;61(1):89-91.

3. Van Der Kleij F, Ziljstra J. Pneumomediastinum and severe subcutaneous emphysema. N Engl J Med 2000;342(18):1333.

4. Kundra M, Yousaf Y, Maqbool S, Mahajan P. Boerhaave Syndrome. Unusual cause of chest pain. Ped Emerg Care 2007;23(7):489-491.
5. Olgun H, Turkilmaz A, Aydin Y, Ceviz N. Spontaneous pneumomediastinum in a child as a rare cause of chest pain. Arch Turk Soc Cardiol. 2009;37(1):51-52.

6. Martín-Menjívar Ernesto, et. Cols. Efecto Macklin como causa de neumomediastino posterior a contusión torácica. Reporte de un caso. TRAUMA. 2007;10(2):58-61.

7. Macklin CC. Transport of air along sheaths of pulmonic blood vessels from alveoli to mediastinum: Clinical implications. Arch Internal Medicine. 1939;64:913-26.

8. Carrillo Esper Raúl, Leal Gaxiola Paul. Pneumomediastino y pneumoperitoneo secundarios a efecto Macklin. Rev Asoc Mex Med Crit y Ter Int. 2008;22(2):111-114.

9. Godoy Laura, Morilla Laura et. Cols. Presentación en la Emergencia de un niño con neumomediastino espontáneo. Pediatr. (Asunción). 2011;38(1):49-52.

10. Cáceres M, Ali SZ, Braud R. Spontaneous pneumomediastinum: a comparative study and review of the literature. Ann Thorac Surg. 2008;86:962-966.

11. Gerazounis M, Athanassiadi K, Kalantzi N, Moustardas M. Spontaneous pneumomediastinum: a rare benign entity. J Thorac Cardiovasc Surg. 2003;126:774-776.

12. Takada K, Matsumoto S, Hiramatsu T. Management of spontaneous pneumomediastinum based on clinical experience of 25 cases. Respir Med. 2008;102:1329-1334.

13. Bilkisa MD, Monteverde E. Efecto Macklin en el neumomediastino espontáneo pediátrico: presentación de casos clínicos. Archargent pediatr. 2010;108(2):33-36.

14. Sakai M, Murayama S, Gibo M et al. Frequent cause of the Macklin effect in spontaneous pneumomediastinum. Demonstration by multidetector row cotomography. J Comput Assist Tomogr. 2006;30:92-94.

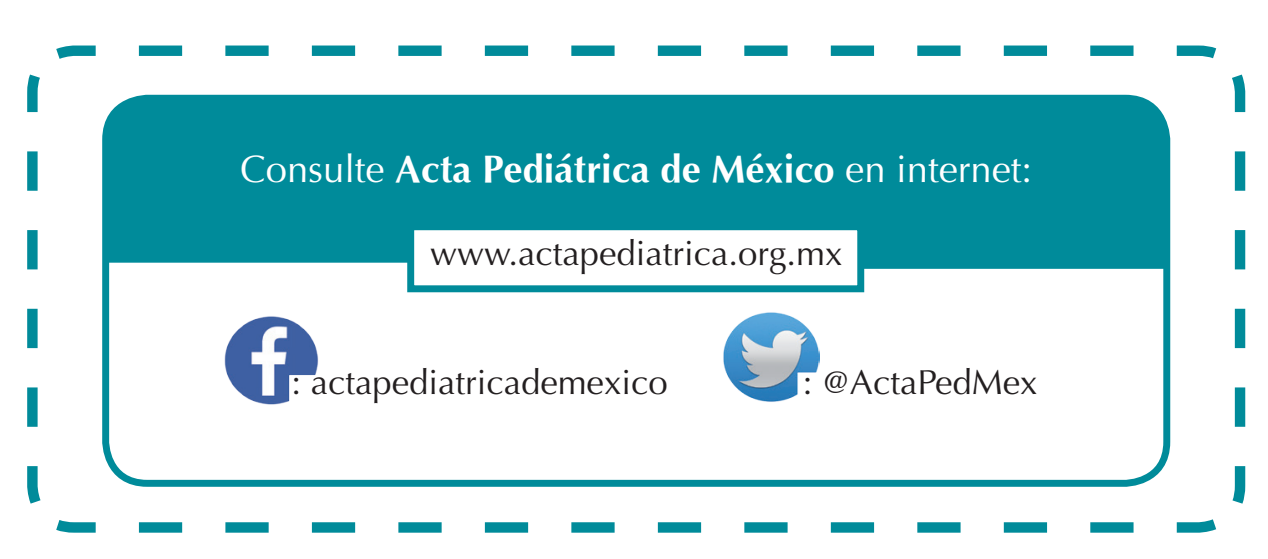

